To appear in Proc. of ICPR 2006

\title{
Template Adaptation based Fingerprint Verification
}

\author{
Choonwoo Ryu, Hakil Kim and Anil K. Jain \\ Michigan State University, BERC, Inha University, Michigan State University \\ choonwoo@cse.msu.edu, hikim@inha.ac.kr,jain@cse.msu.edu
}

\begin{abstract}
This paper proposes a minutiae-based template adaptation algorithm which can be applied after the fingerprint authentication process. The algorithm updates a template by using a query fingerprint, which is successfully verified by the fingerprint matcher as a high quality genuine input. This algorithm generates an updated minutiae set by using not only the minutiae but also local fingerprint quality information and utilizes a successive Bayesian estimation to evaluate the credibility of minutiae and their types. The proposed algorithm updates fingerprint minutiae information in the template as well as appends new minutiae from the query fingerprint. Preliminary experiments show an average $32.7 \%$ EER reduction and an even higher matching accuracy improvement at low false accept rates.
\end{abstract}

\section{Introduction}

Fingerprint verification systems have been widely accepted in the market due to their superior authentication accuracy and availability of compact and reasonable price sensors. Due to increasing demands on automatic fingerprint technology, a lot of research has been done on fingerprint verification techniques. However, many problems still remain because of the nature of fingerprints or low-end fingerprint acquisition systems. For example, low quality fingerprint images lead to less reliable fingerprint features on account of various fingerprint conditions (e.g. dry or wet fingers) or the surface condition of the fingerprint scanner.

In order to overcome the limited performance of the fingerprint feature extraction from a single fingerprint, multi-impression approaches [1-5] have been proposed for the purpose of creating a template from multiple fingerprint images during enrollment. These multiimpression approaches can be distinguished based on the information source that is used, i.e., images or features. The image-based multi-impression approaches utilize minutiae for coarse alignment between multiple images. After coarse alignment, Jain et al. [1] modified an iterative closest point algorithm, while Lee et al. [2] suggested a distance map matching for fine alignment.

Most of the feature-based approaches [3-5] utilize minutiae as the feature and generate a template at the time of enrollment. Ramoser et al. [3] and Yau [4] merged corresponding minutiae into a single minutia but no minutiae evaluation is made. Therefore, all the minutiae found in the feature extraction are assumed to be the true minutiae, which means that all false minutiae will be included in the template. The supertemplate generation approach [5], however, conducts minutiae evaluation that removes low quality minutiae and changes minutiae type according to the type credibility.

Generating a superior template using multiple fingerprint images at enrollment is not sufficient for the long-term satisfactory operation of a fingerprint verification system. Several factors can change a system's performance over time, i.e., the user's finger surface, user habituation or circumstance of the fingerprint system. Figure 1 shows an example of finger surface condition change. Comparing the two images in Figure 1, sensor condition and user habituation seem to be similar but the fingerprint in Figure 1(b) has scars and an unclear ridge and valley structure region due to finger skin being peeled off.

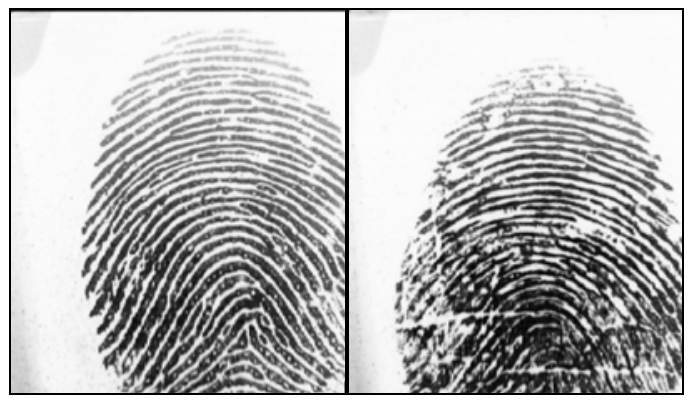

(a) First day fingerprint

(b) After 100 days

Figure 1. Change of a finger surface condition 
Template adaptation is one approach to handle temporal changes in fingerprint images. Jiang et al. [6] improved the credibility of the minutiae by applying fusion techniques only after successful genuine matchings. This approach uses minutiae and fingerprint segmentation information, where reliable minutiae are selected by estimating minutia credibility using a fixed weighting recursive function. According to the fixed weighting nature, the number of occurrences of a minutia at the same location will directly decide the minutia credibility. No new minutiae from the query can be appended if the minutiae are found in the background of the initial template segmentation. Hence, fingerprint matching in a small-size sensor may produce a high false reject rate.

This approach is different from our proposed algorithm. We not only update minutiae information in the template but also append all new minutiae that are allowed in any location in the query image. Therefore, the fingerprint area of the template can increase by adapting more query images. The algorithm generates an updated minutiae set by using not only minutiae but also local fingerprint quality information and utilizes a successive Bayesian estimation (SBE) in order to evaluate the credibility of each minutia and its type (i.e., ridge ending or bifurcation). The local fingerprint quality is employed to estimate the probability that the minutia and its type are true. Then, SBE updates the probability effectively and adaptively based on the quality.

The details of the proposed template adaptation algorithm are explained in section 2. Section 3 shows experimental results, followed by the conclusions in Section 4.

\section{Template adaptation}

Figure 2 shows an overview of the proposed template adaptation algorithm. Minutiae in query are extracted by a feature extraction algorithm [7]. The quality map in query is the local fingerprint quality in blocks of size $w \times w$. Using gray level gradient vectors $[d x d y]^{T}$ of all the pixels in a block, the local quality of each block centered at pixel $(i, j)$ is defined as follows:

$$
Q(i, j)=\left\{\begin{array}{cc}
0 & \text { background } \\
1-\frac{(a+b)-\sqrt{(a-b)^{2}+4 c^{2}}}{(a+b)+\sqrt{(a-b)^{2}+4 c^{2}}} & \text { otherwise }
\end{array}\right.
$$

where $a=E\left\{d x^{2}\right\}, b=E\left\{d x^{2}\right\}$ and $c=E\{d x d y\}$. This is a modified Principle Component Analysis approach for local fingerprint quality [8]. $Q(i, j)$ is assigned 0 when the point $(i, j)$ is located in the fingerprint background; otherwise, the quality value equals ( 1 - eigenvalue ratio). $Q(i, j)$ is between 0 and 1 , where a value of 1 indicates the highest fingerprint quality.

Our template updating algorithm maintains two sets, minutiae and minutiae candidate, to store minutiae information. Minutiae having higher credibility than a certain threshold belong to the minutiae block and the rest are stored in the minutiae candidate block. The minutiae block is used for actual matching against the block in a query, while the minutiae candidate is used for minutiae updating. After template adaptation, the minutiae in minutiae candidate having high credibility move to minutiae while minutiae with low credibility are moved from minutiae to minutiae candidate. The quality map in the template corresponds to quality maps which can be decided by a number of last images or images in the last $k$ days. The maps will be utilized in estimating the credibility of a new minutia from a query.

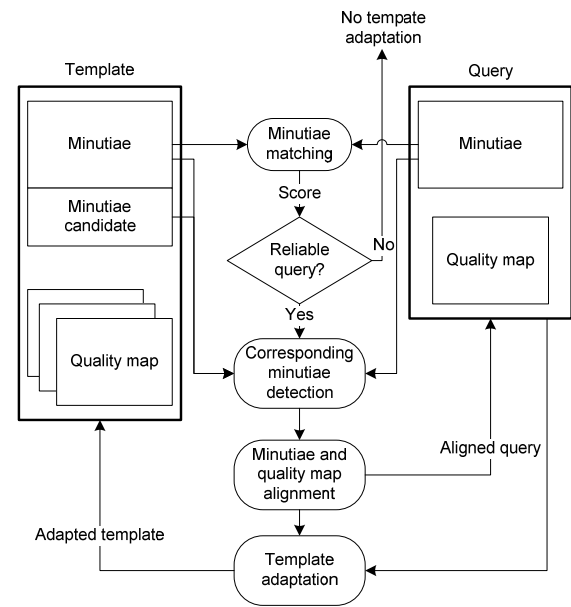

Figure 2. Template adaptation approach

In this study, a minutiae matcher calculates the matching score using an elastic point matching algorithm [7]. If the matching score is above a given threshold, then template adaptation will be performed. Otherwise, the template will be preserved. Before the template adaptation, corresponding minutiae are found between the union of minutiae and minutiae candidate and the minutiae in the query by the same matcher that gives the matching score. The corresponding minutiae in the template will be updated by the minutiae information in the query.

Using the corresponding minutiae, this study determines a transformation parameter which delivers the best query transformation to the template in the same coordinate space. Most of the fingerprint matchers use a transformation function and compute its parameter; which is reasonable because the matchers compute not a globally optimized parameter but a locally well matched parameter of the function using a 
small number of pairs of minutiae, called local structure. Even the best parameter among the local structure pairs may not satisfy the transformation of the corresponding minutiae because fingerprint matchers, like our matcher in this study, may search corresponding minutiae under the consideration of fingerprint non-linear deformation nature.

In this study, the linear transformation described in Eq. (2) is defined for the transformation function of the query to template in the same coordinate space.

$$
\left[\begin{array}{l}
x_{t} \\
y_{t}
\end{array}\right]=\left[\begin{array}{lll}
t_{11} & t_{12} & t_{13} \\
t_{21} & t_{22} & t_{23}
\end{array}\right]\left[\begin{array}{c}
x_{q} \\
y_{q} \\
1
\end{array}\right]
$$

The vector $\left[\begin{array}{ll}x_{t} & y_{t}\end{array}\right]^{T}$ is a minutia position in the template, and $\left[\begin{array}{lll}x_{q} & y_{q} & 1\end{array}\right]^{T}$ is the corresponding minutia coordinate of $\left[\begin{array}{ll}x_{t} & y_{t}\end{array}\right]^{T}$ in the query. At least three non-collinear corresponding points are required to solve the transformation matrix $T=\left\lfloor t_{i j}\right\rfloor$. In this study, all corresponding minutiae points are used to decide the matrix $T$ in the sense of the least mean square error. After computing the matrix $T$, minutiae and quality map in the query are transformed by the matrix $T$ to the template coordinate space. The aligned quality map will be registered into the template after all minutiae are updated.

Probability that the feature extraction will find the true at a certain location $(x, y)$ is a function of local quality $Q(x, y)$. It is sensible because genuine minutiae are produced in good quality image areas while false minutiae are more likely generated from poor quality image areas. The probability function can be designed by analyzing the results of feature extraction over different image quality areas. In this study, we simplify the function as

$$
p_{m}=p[Q(x, y) \mid m]=\left\{\begin{array}{cc}
0.5 & \text { background } \\
\alpha Q(x, y)+\beta & \text { otherwise }
\end{array}\right.
$$

where $\alpha+\beta \leq 1, \alpha, \beta \geq 0$ and $p_{m}=0.5$ when minutiae location is in the fingerprint background. This function can vary depending on the feature extraction performance and the definition of local quality. In this manner, the probability that the minutia type is true, $p_{\zeta}$, can be defined as the same form.

Credibility of a minutia in the template is recursively estimated by using SBE. The minutia credibility $p_{m c}$ is updated by the current measurement as described in Eq. (4), where $\{Q\}_{N}$ represents the set of entire measurement of local quality at the minutia location until $N$ previous queries. The updating rule reinforces the probability that the minutia is genuine from the past values based on the current measurement.

$$
p_{m c}^{\prime}=p\left[m=\text { true } \mid\{Q\}_{N}\right]=\frac{p\left[Q_{N} \mid m=\text { true }\right] p\left[m=\text { true } \mid\{Q\}_{N-1}\right]}{\sum_{m} p\left[Q_{N} \mid m\right] p\left[m \mid\{Q\}_{N-1}\right]}
$$

In this manner, minutia type credibility $p_{\zeta c}$ can be evaluated. However, the minutia type can be switched after credibility updating when the credibility is below 0.5. For example, if the type is ridge ending and the credibility $p_{\zeta c}$ becomes 0.3 by the updating scheme then the type will become bifurcation and its credibility will be 0.7 .

Minutiae in template adaptation can be divided into three types: corresponding minutiae $\left(m_{c p}\right)$, minutiae only in the template ( $\left.m_{o t}\right)$ and minutiae only in the query $\left(m_{o q}\right)$. Minutiae $m_{o q}$ are registered in the template with the same minutia locations and directions as the minutiae in the query. However, the locations and directions of minutiae $m_{c p}$ are adapted by the corresponding minutiae in the query. It is described as

$$
\begin{aligned}
& \left(x_{t}^{\prime}, y_{t}^{\prime}, \theta_{t}^{\prime}\right) \\
& =\left(\frac{x_{t} p_{m c}+\gamma x_{q} p_{m}}{p_{m c}+\not p_{m}}, \frac{y_{t} p_{m c}+\not y_{q} p_{m}}{p_{m c}+p_{m}}, \tan ^{-1}\left(\frac{\sin \left(\theta_{t}\right) p_{m c}+\gamma \sin \left(\theta_{q}\right) p_{m}}{\cos \left(\theta_{t}\right) p_{m c}+\gamma \cos \left(\theta_{q}\right) p_{m}}\right)\right)
\end{aligned}
$$

where $\gamma$ is a constant value for scaling the query.

Credibility $p_{m c}$ of the minutiae $m_{c p}$ and $m_{o t}$ is adapted by Eq. (4) with $p\left[m=t r u e \mid\{Q\}_{N-1}\right]$, i.e. output of Eq. (4) at the $(N-1)$ th measurement and $p\left[Q_{N} \mid m\right]$ (or $1-p\left[Q_{N} \mid m\right]$ for $m_{o t}$ ) from Eq. (3). However, minutiae $m_{o q}$ do not have the probabilities $p\left[m=\right.$ true $\left.\mid\{Q\}_{N-1}\right]$ because the minutiae do not exist in the template until $N$-1. In this case, the initial probabilities $p\left[m=t r u e \mid\{Q\}_{0}\right]$ are generated before applying the updating rule. Using average quality of the quality maps in the template, the initial credibility is given as

$$
p\left[m=\operatorname{true} \mid\{Q\}_{0}\right]=1-p\left[\frac{1}{n} \sum Q(x, y) \mid m\right]
$$

where $Q(x, y)$ denotes the quality map in the template and $n$ represents the number of quality maps where the location $(x, y)$ is in the foreground. Equation (6) describes the probability that a feature extractor misses a minutia under the given average image quality condition. In this manner, the credibility $p_{\zeta c}$ is updated.

\section{Experimental results}

The database for the experiments was collected at Inha University, Korea. The database contains 41 subjects whose fingerprint images were captured over a period of 100 days. These subjects are undergraduate students who provided their fingerprint for the purpose of marking their attendance in a lecture during one semester. On the first day of the lecture, they registered their fingerprint into the system under supervision. Each time they attended the class thereafter the students provided their fingerprint in order to verify 
their attendance. Therefore, the volunteers are cooperative.

To simulate template adaptation in real systems, the first image of the each subject is assigned as a template and the other images are assigned as a query. This is reasonable because a user gains access to the system after registering his biometrics data. In our experiments, the first image never becomes a query image even in impostor matching.

Template adaptation changes the previous template; therefore, the next matching result relies on the previous matching sequence. Even if the previous matching pairs are the same but the sequence is different, then the matching score of the next matching may be different. As a result, this study performs a conventional matching and three different matching sequences [9] for template adaptation.

As shown in Table 1, conventional matching ("No update") has 3.84\% EER (Equal Error Rate) but the template adaptation matching drops the average EER (average of three template adaptation matching) to $2.58 \%$. This demonstrates a $32.7 \%$ EER reduction, on average. More than $50 \%$ of error reductions are observed at ZeroFMR and ZeroFNMR.

Table 1. Selected experimental results

\begin{tabular}{|l|l|l|l|l|}
\hline \multirow{2}{*}{$\begin{array}{c}\text { Performance } \\
\text { indicator }\end{array}$} & \multirow{2}{*}{$\begin{array}{c}\text { No } \\
\text { update }\end{array}$} & $\begin{array}{c}\text { Impostor } \\
\text { first }\end{array}$ & $\begin{array}{c}\text { Random } \\
\text { matching }\end{array}$ & $\begin{array}{c}\text { Genuine } \\
\text { first }\end{array}$ \\
\hline EER & $3.84 \%$ & $2.47 \%$ & $2.48 \%$ & $2.80 \%$ \\
\hline ZeroFMR & $37.32 \%$ & $21.68 \%$ & $10.39 \%$ & $15.87 \%$ \\
\hline ZeroFNMR & $68.25 \%$ & $30.06 \%$ & $33.24 \%$ & $38.57 \%$ \\
\hline
\end{tabular}

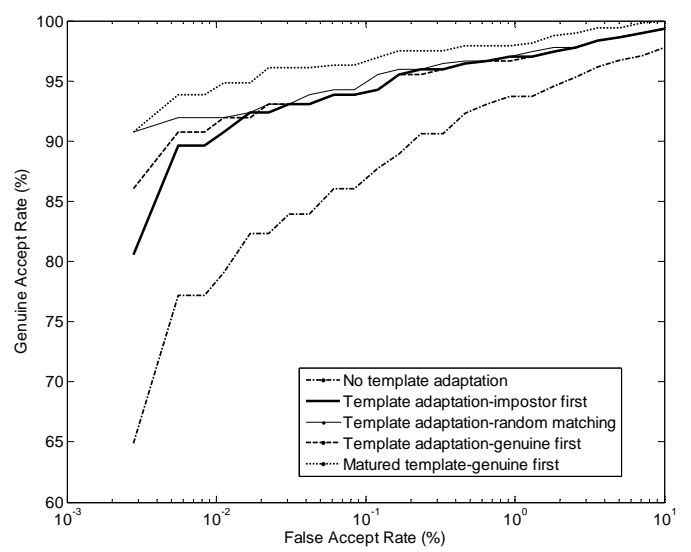

\section{Figure 3. ROC showing performance improvement in template adapted matching}

Figure 3 shows ROC curves of each experiment. All the template adaptation matchings have higher GAR (Genuine Accept Rate) than matching with no adaptation. Performance degradation is not as severe as with conventional matching because the accuracy curves in all template adaptations have small slopes. The proposed algorithm provides the same user convenience, i.e. same GAR, at a security level that is about 10 times higher. After the templates become matured by adapting 10 images, matching accuracy is improved as $1.75 \%$ EER and has higher GAR for all FAR values as shown in Figure 3.

\section{Conclusions}

In this study, we have proposed a minutiae-based template adaptation algorithm and applied it to a minutiae-based feature extraction and matching algorithm. The proposed algorithm not only updates minutiae in a template by using a query but also includes new minutiae from the query. This is an attractive feature for small-sized sensors, which have authentication weakness due to the small sensing area. The proposed algorithm evaluates minutia credibility in a template by utilizing a successive Bayesian estimation with local fingerprint quality, which updates minutiae adaptively. Experimental results demonstrate $32.7 \%$ EER reduction and even higher error reduction at low value of FAR.

\section{References}

[1] A. K. Jain and A. Ross, "Fingerprint Mosaicking," Proc. Int'l Conf. on Acoustic Speech and Signal Processing, vol 4. pp. 4064-4067, 2002.

[2] D. Lee, K. Choi, S. Lee and J. Kim, "Fingerprint Fusion Based on Minutiae and Ridge for Enrollment," Proc. AVBPA 2003, LNCS 2688, pp. 478-485, 2003.

[3] H. Ramoser, B. Wachmann and H. Bischof, "Efficient alignment of fingerprint images," Proc. Int'l Conf. on Pattern Recognition, pp. 748-751, 2002.

[4] W. Yau, K. Toh and T. Chen, "Fingerprint Templates Combination," Proc. 5th Chinese Conference on Biometric Recognition, LNCS 3338, pp. 449-460, 2004.

[5] C. Ryu, Y. Han and H. Kim, "Super-template Generation Using Successive Bayesian Estimation for Fingerprint Enrollment,” Proc. AVBPA 2005, pp. 710-719, 2005.

[6] X. Jiang and W. Ser, "Online Fingerprint Template Improvement,” IEEE Trans. PAMI, vol. 24, no. 8, pp. 11211126, August 2002.

[7] A.K. Jain, L. Hong and R. Bolle, “On-line Fingerprint Verification,” IEEE Trans. on PAMI, vol. 19, no. 4, pp. 302314, 1997.

[8] E. Lim, X. Jiang and W. Yau, "Fingerprint Quality and Validity Analysis,” Proc. of Int'l Conf. on Image Processing, vol. 1, pp. I-469 - I472, September 22-25, 2002.

[9] C. Ryu and H. Kim, "Fingerprint Verification Testing Scenarios for Multi-impression Enrollment and Template Adaptation," Proc. of Biometric Symposium, pp. 39-40, September 19-21, 2005. 